\title{
Multi-flavour PeV neutrino search with IceCube
}

\section{The IceCube Collaboration ${ }^{\dagger}$}

$\dagger$ http://icecube.wisc.edu/collaboration/authors/icrc17_icecube

E-mail: lu.ludicecube.wisc.edu

The IceCube observatory, located at the South Pole has been completed in 2010 and is the largest neutrino detector in the world. PeV neutrinos have been discovered in previous analyses which were optimised for different event topologies. A new search has been developed to select PeV cascades that are not fully contained in the detector. It is therefore sensitive to the Glashow resonance at $6.3 \mathrm{PeV}$. Together with the existing high-energy-starting-events (HESE) and extremehigh-energy (EHE) channels, the combined event selection provides the highest sensitivity to the beyond-PeV region, where a cut-off in the spectrum could occur. We will show the sensitivities for six years of data taking to demonstrate the preferred scenario for the cut-off energies and possible constraints on the flavour-dependent cut-off. The result is important both for understanding the source properties and for providing the possibility of reliable astrophysical-background estimations of GZK neutrino searches.

Corresponding authors: L. Lu*1

${ }^{1}$ Department of Physics, Chiba University, Chiba 263-8522, Japan

35th International Cosmic Ray Conference - ICRC2017

10-20 July, 2017

Bexco, Busan, Korea

${ }^{*}$ Speaker. 


\section{Introduction}

After more than a century since the discovery of cosmic-rays, we know little about their origins. These charged particles carry extreme energies and are expected to interact with gas and radiation at the source, producing pions which subsequently decay into neutrinos and photons. The fact that neutrinos are weakly-interactive makes them ideal messengers for studying the highenergy universe but also makes them hard to be detected. One example of indirect measurement of neutrinos is to detect Cherenkov photons, which are emitted by charged particles produced in the interactions between neutrinos and matter in a transparent medium such as in water or ice.

The IceCube Neutrino Observatory, located at the South Pole, was completed in 2010 with more than 5000 photo-sensors buried in the deep ice that instrument a volume of one cubickilometre to search for such Cherenkov photons. The detection of extraterrestrial neutrinos has been reported in [1] and in particular four neutrinos with deposited energy greater than $1 \mathrm{PeV}$ have been found $[2,3,4]$. The discoveries by the IceCube collaboration opened a new window for multi-messenger astronomy that allows to probe the cosmic-ray sources and their properties via the neutrino channel. For instance, the energy of the neutrinos is expected to be $\sim 5 \%$ of the energy of the nucleon at the source. By measuring a cut-off in the energy spectrum of the neutrinos one could learn about the acceleration capability of the source. More importantly, a significant constraint on the existence of a spectral cut-off at high energies would provide information on whether the observed IceCube spectrum extends beyond few $\mathrm{PeV}$, and if it might be connected with the sources of ultra-high energy cosmic-rays.

The Glashow resonance [5] at $6.3 \mathrm{PeV}$ enhances the detection probability for electron antineutrinos and could be used to constrain a potential spectral cut-off in the PeV energy range. In this paper, we introduce a new event selection that was developed to recover $\mathrm{PeV}$ events which failed the selection criteria of both, the high-energy starting event sample (HESE) [2] and the extremehigh energy event sample for on-line alerts (EHE-PeV) [1,7]. The sensitivity for constraining a cut-off with all three combined sample is shown for 6 years of data-taking.

\section{A new channel for PeV neutrinos}

Among the four multi-PeV events seen so far by IceCube, three are cascades with vertex positions inside the detector volume and one is a through-going track. Several analyses targeting different physics goals $[1,2,4,8]$ have detected these events independently. The HESE sample relies on a veto technique [2] to efficiently reject cosmic-ray background and only selects events with vertex positions located within the fiducial volume. The EHE-PeV sample selects throughgoing tracks induced by $v_{\mu}$ with energies $\sim 1 \mathrm{PeV}$. To illustrate the characteristics of electron neutrinos selected by HESE, Figure 1(a) shows the vertex position of HESE-selected $v_{e}$ events of energies greater than $1 \mathrm{PeV}$. Figure 1(b) shows events which are selected neither by HESE nor by EHE-PeV.

As expected, the HESE selection is limited by the fiducial volume. The gap at $z \approx-200 \mathrm{~m}$ indicates the position of the dust layer in the ice, which is known to feature strong absorption and scattering of light. High-energy events often register significant amount of signals in the detector but with a vertex position at or outside of the detector boundary. It is evident that to increase the 


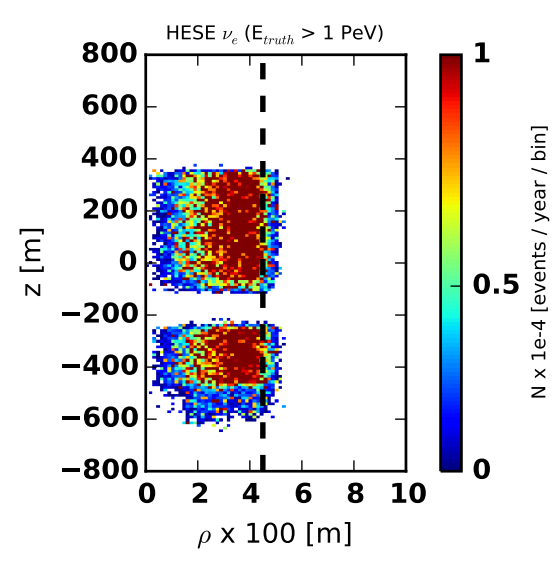

(a)

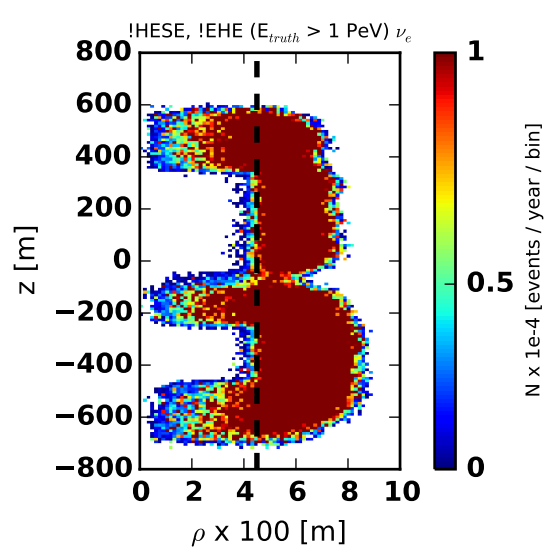

(b)

Figure 1: (a) Vertex positions of simulated multi-PeV electron neutrinos selected by HESE. (b) Vertex positions for those which failed the selection criteria of both, HESE and EHE-PeV. The colour scale indicates the event rate per bin. $\rho, z$ denote cylindrical coordinates with the origin in the center of IceCube. The neutrino spectrum is assumed to be $E^{-2}$ for neutrinos with $v_{e} / \bar{v}_{e}=1: 1$.

effective volume of the HESE sample, one needs to extend the allowed region for the interaction vertex position to the outside of the detector. The goal of this analysis is therefore the selection of PeV Energy Partially-contained Events (PEPE), with special focus on the Glashow resonance at 6.3 PeV. The dust-layer is also excluded in the new event selection.

Since the branching ratio of the $W^{-}$decay is $\sim 67 \%$ for hadron jets and $\sim 11 \%$ for electromagnetic showers, the dominant Glashow signals are cascade-like events. A previous study from IceCube [8] already explored the possibility of including the partially-contained cascade channel using a simple classification of the events based on their reconstructed properties. Here we propose to use machine-learning techniques to further enhance the classification of signal and background with emphasis on the signal purity at the energy of the Glashow resonance.

There are $>10^{7}$ events generating at least $10^{3}$ photoelectrons in the ice per year although only few of which are expected to be PeV neutrino signals. The majority of the events are track-like background from muons produced by cosmic rays propagating in the atmosphere. To ensure the new event sample is statistically independent from the HESE and EHE-PeV samples, the events that pass the former two selections are excluded from the PEPE sample. Next a gradient-boosted decision tree (BDT) [9] was trained with 11 observables such as the rise time of the PMT waveform, and the displacement of the mean position of the detected photons from the interaction vertex. Note that none of the BDT inputs needs high-level reconstructions, which reduces the effects due to uncertainties on the ice properties. Only electron neutrinos of generated energies $>1 \mathrm{PeV}$ are labelled as signal in the training. Selection efficiencies and expected rates for signal and background at various steps in the selection process is shown in Figure 2.

Approximately $\sim 20 \%$ of the electron neutrinos are selected by HESE, leaving the remaining of $\sim 80 \%$ unexplored. By selecting events of BDT score $>0.5, \sim 40 \%$ of the PeV signals are saved by PEPE. The remaining $40 \%$ are rejected due to being located in the dust layer or having vertex positions too far away from the edge of the detector to be identified as signal. The events 


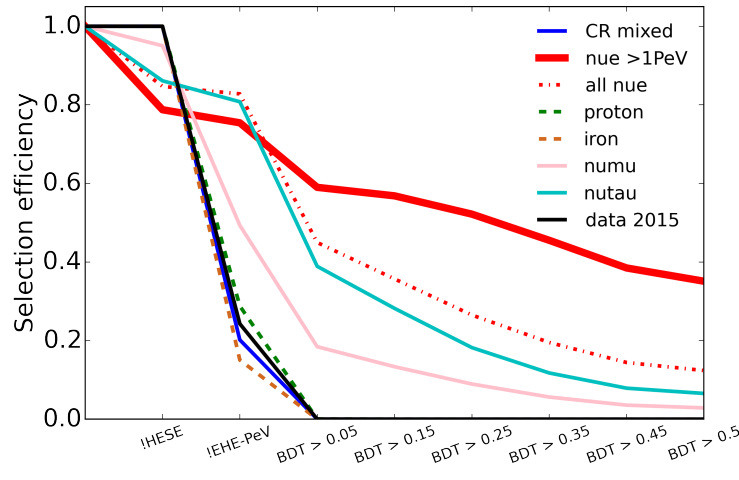

(a)

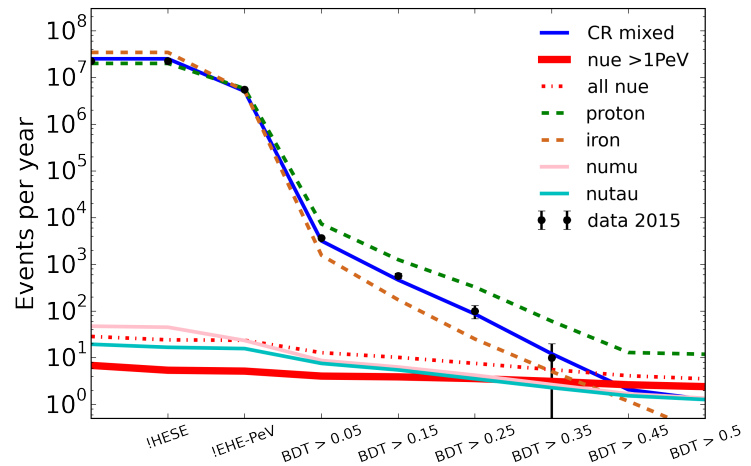

(b)

Figure 2: Decrease of selection efficiency and event rate due to HESE, EHE-PeV and BDT score requirements. $10 \%$ of data took in 2015 is shown in black to compare with simulations. The solid red lines are expectations of electron neutrinos of energy greater than $1 \mathrm{PeV}$ under the assumption of an $E^{-2}$ spectrum.

with vertex position more than a string-spacing away from the outermost layer of the detector are less bright, harder to be distinguished from background, and more difficult to reconstruct. An illustration of events kept or rejected by the BDT cut is shown in Figure 3.

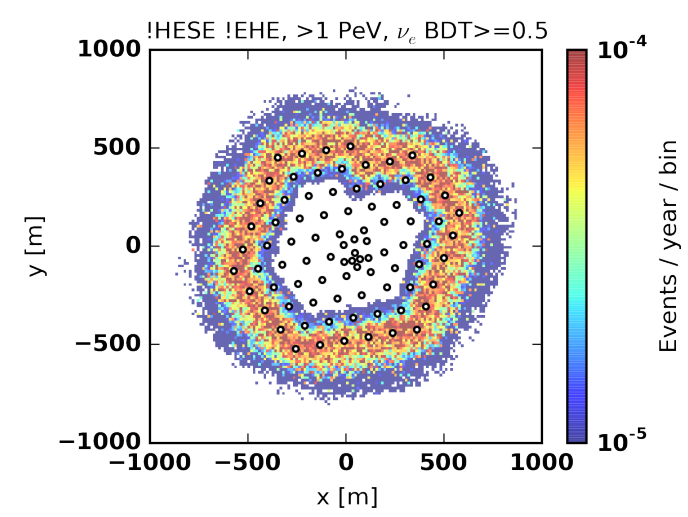

(a)

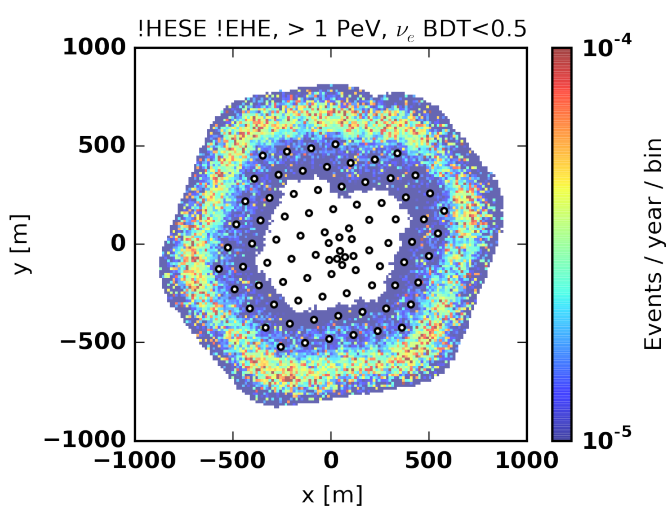

(b)

Figure 3: (a) X-Y projection for vertex positions of electron neutrinos with energy $>1 \mathrm{PeV}$ and BDT score $>0.5$ (b) Vertex positions for electron neutrinos of energy $>1 \mathrm{PeV}$ and BDT score $<0.5$

For cosmic-ray background, the BDT score is found to be correlated with the fraction of the energy carried by the leading muon over the total energy of all muons reaching IceCube. Highenergy muons lose energy stochastically and could register as cascade-like signals when the track travels outside of the detector. Cosmic-rays with BDT score $>0.5$ are mostly high-energy leading muons of energy $\sim \mathrm{PeV}$ carrying $>90 \%$ of the total muon signal in the shower. These rare events are dominantly produced in the air-showers originating from cosmic-ray protons. Simulations were generated via CORSIKA [10] with SIBYLL2.3 [11], charm components have been included. The dominant contribution is from kaons and pions and the charm fraction is less than $10 \%$ for leading muons with energies of $\sim 1 \mathrm{PeV}$. 
To reconstruct cascade-like events, a point-like Cherenkov light emitter is assumed and the expectations of the number of Cherenkov photons and their arrival times at each DOM is compared to observations or simulated events. Photons from the tracks that comprise the background have worse agreement with such a hypothesis than photons from cascades. This allows further reduction of the background using a goodness-of-fit estimator. The final event selection parameters are optimised for the best data-MC agreement in the background-dominated region and the best signal-background separation in the signal-dominated region, defined by a BDT score $>0.5$ and a goodness-of-fit $<10$.

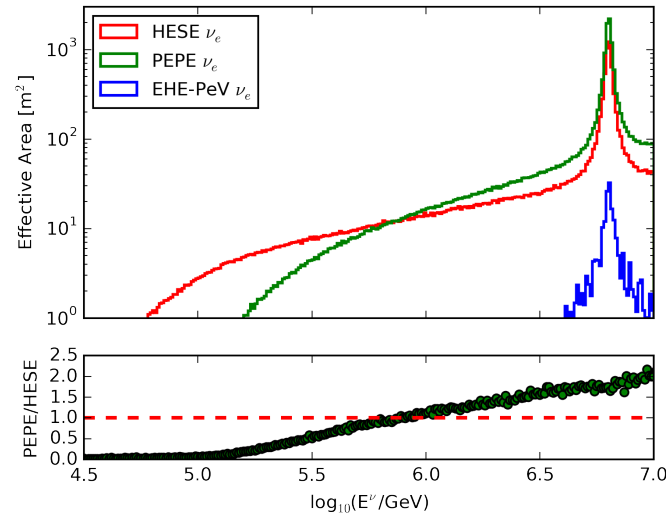

(a)

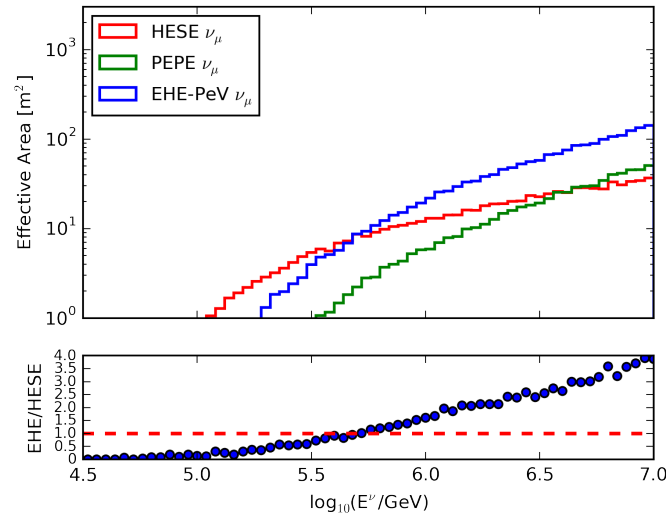

(b)

Figure 4: Effective area for $v_{e}$ (a) and $v_{\mu}$ (b) of HESE, PEPE and EHE-PeV

The effective areas averaged over solid angle of $v_{e}$ and $v_{\mu}$ are shown in Figure 4. The PEPE sample surpasses HESE at $\sim 500 \mathrm{TeV}$ for detecting electron neutrinos and the gain increases with neutrino energy.

\section{Event reconstruction of partially-contained cascades}

The reconstruction of partially-contained cascades is based on a likelihood fit which maximises the agreement between observation and expectation for the arrival time and intensity of Cherenkov photons assuming a cascade of energy E, vertex position (x,y,z), zenith angle $\theta$ and azimuth angle $\phi$. To understand how well partially-contained cascades can be reconstructed and how well data agrees with simulations, one of the PeV contained cascades detected by IceCube [1] has been selected for a cross-check with simulations. A thousand PeV-cascade events are simulated with vertex positions in a sphere centred at the best-fit value and a radius corresponding to the uncertainty of the vertex position fit. The energy is set to the best-fit energy. For each of the simulated events, the same reconstruction algorithms are applied, and the reconstructed energy and the zenith angle are recorded. Simulations are made using the SpiceLea model for the optical properties of the ice [12], which takes into account the anisotropy of the light propagation and the tilt of the ice layers. The distribution of $\Delta E / E$, which is defined as $\left(E_{\mathrm{rec}}-E_{\mathrm{MC}}\right) / E_{\mathrm{MC}}$ is shown in Figure 5(b).

In the next step, a wall of strings from the edge of the detector is artificially removed from the reconstruction process for both data and simulations, as illustrated in Figure 5(a). It was found 


\begin{tabular}{lcccc}
\hline Config. & removed string count & NPE & $\Delta \mathbf{E} / \mathbf{E}(\mathrm{MC})$ & $\Delta \mathbf{E} / \mathbf{E}$ (data) \\
\hline original & N/A & 24830 & $0.6 \%$ & $0 \%$ \\
-1 layer & 6 & 24707 & $0.5 \%$ & $-0.2 \%$ \\
-2 layers & 13 & 21854 & $-1.9 \%$ & $-4.9 \%$ \\
-3 layers & 21 & 8587 & $30 \%$ & $25 \%$ \\
\hline
\end{tabular}

Table 1: Comparison of the following configurations: the unmodified reconstruction of the measured PeVevent and three modified reconstructions with less layers of strings (see text). The number of photoelectrons (NPE), the difference in reconstructed energy, $\Delta E / E$ for simulations and data, are given.

that the effect of reconstruction for the real data yields $\left(E_{\text {cut } 1 \text { layer }}-E_{\text {nocut }}\right) / E_{\text {nocut }}$ about $-0.2 \%$ and a bias on reconstructed zenith angle of about $-0.5^{\circ}$, which are in good agreement with the simulation.

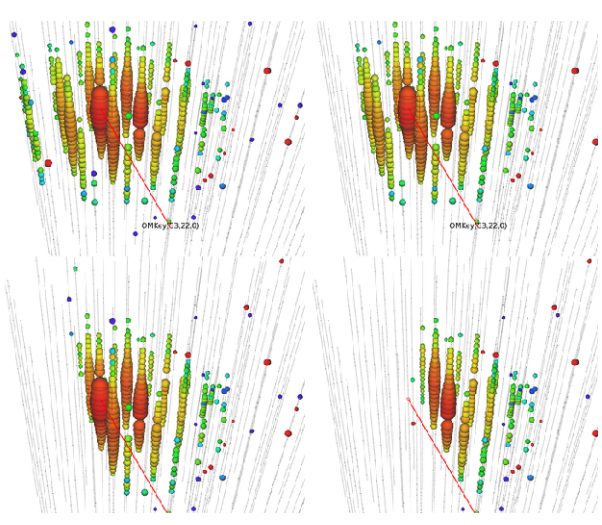

(a)

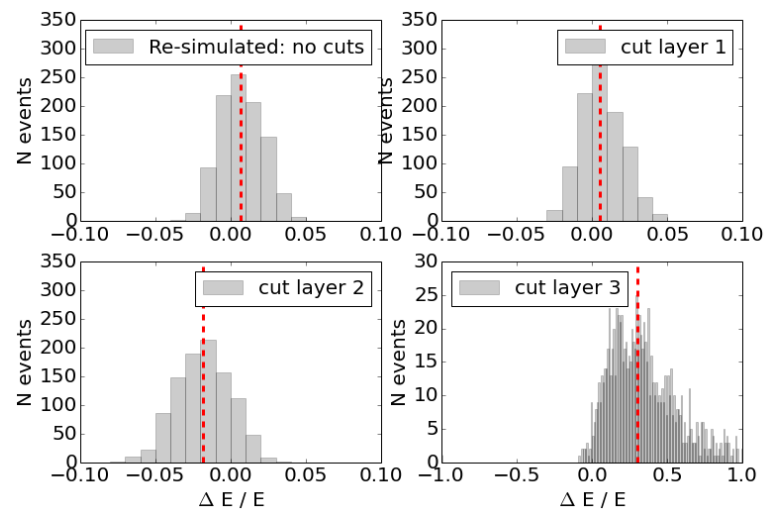

(b)

Figure 5: (a) Event view of one of the contained PeV events found by IceCube and after one, two and three outer layers of strings have been removed. (b) Reconstruction resolutions of energy for re-simulated PeV events. Each histogram corresponds to 1000 events simulated in one of the scenario in (a) and the dashed lines show the median of each distribution. The reconstructed results from the real data is shown in Table1.

Further removal of a layer of strings results in $-5 \%$ energy bias and $+0.35^{\circ}$ of reconstructed zenith angle for data. With three layers of strings removed, the event is left with $1 / 3$ of total charge, the biases of reconstructed energy and zenith angle are $+25 \%$ and $+20^{\circ}$. All the cases data and simulations agree within allowed uncertainty range. The overestimation of energy in the latter case is due to a wrongly fitted vertex position. The best-fit vertex positions are dragged further outside of the detector, so to compensate the signals the energy of the event needs to be higher. More tests have been carried out such as to remove DOMs on horizontal layers. The same conclusion is reached: when the vertex position is located far outside of the detector and only a fraction of scattered photons are observed, the reconstructed energy can be overestimated. The bias has been observed consistently for both data and simulations. It is planned to do further studies to improve the reconstruction on an event-by-event basis if $\mathrm{PeV}$ events are found in the data. For events selected by PEPE, the median error of zenith angle is $\sim 15^{\circ}$ and the median error on deposited energy is within $20 \%$. 


\section{A global fit for a spectral cut-off at PeV energies}

A fit of the spectral parameters is performed using a binned Possion-likelihood method comparing the observed and expected number of events for two bins in zenith angle and five bins per decade in reconstructed energy. Track-like events from the HESE sample are not included in this fit, but only events which can be reconstructed with a single point-like cascade hypothesis are included.

About a factor of two more Glashow resonance events are expected in the PEPE sample when compared to the HESE sample. Similarly the EHE-PeV selection nearly doubles the sensitivity for PeV $v_{\mu}$ compared to HESE. The combined dataset allows to test the presence of a cut-off and its energy more precisely than with the HESE sample alone. An Asimov-style [13] test has been carried out with the following assumptions: a 1:1:1 flavour mixing ratio, $v_{e}: \bar{v}_{e}=1: 1$ and a neutrino spectrum of $\mathrm{d} \Phi / \mathrm{d} E=2.7 \times(E / 100 \mathrm{TeV})^{-2.29} e^{-E / 2.21 \mathrm{PeV}} \times 10^{-18} \mathrm{GeV}^{-1} \mathrm{~cm}^{-2} \mathrm{~s}^{-1}$, which is consistent with the best fit result from the global analysis [14]. The profile-likelihood scan on the spectral index $\gamma$ and the cut-off energy is shown in Figure 6. The contours have been obtained assuming Wilks' theorem applies [15]. No systematic uncertainties are taken into account at this stage.

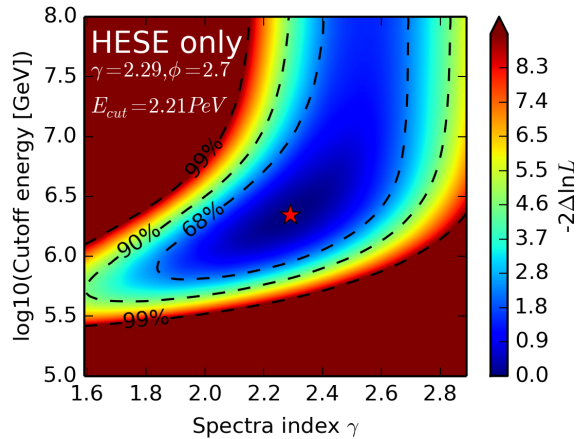

(a)

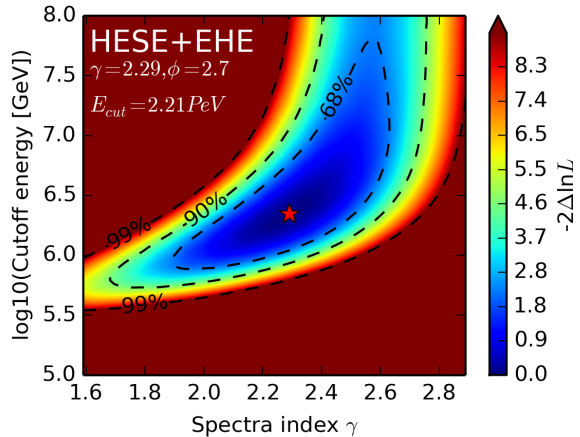

(b)

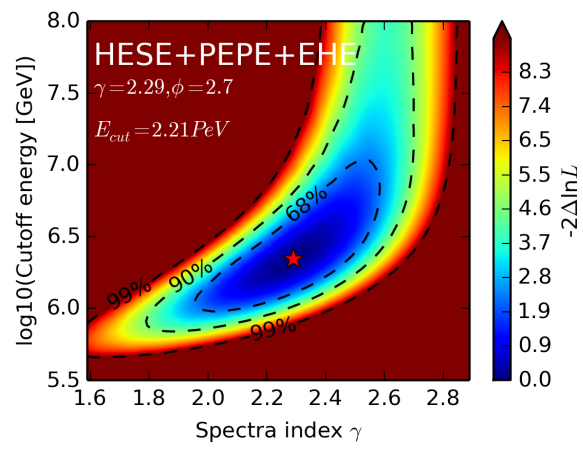

(c)

Figure 6: Profile likelihood scan on $\gamma$ and cut-off energy for Asimov test of $\gamma=-2.29$, normalisation $=2.7 \times$ $10^{-18} \mathrm{GeV}^{-1} \mathrm{~cm}^{-2} \mathrm{~s}^{-1}$ and $E_{\mathrm{cut}}=2.2 \mathrm{PeV}$ (a) HESE only contour (b) HESE+EHE contour (c) combined HESE+EHE+PEPE.

The prediction of the event rate at the Glashow resonance energy depends greatly on the cut- 
off energy. For instance, assuming a hard spectral index $\gamma=-2.09$, the number of events expected in 6 years with reconstructed energy above $2.5 \mathrm{PeV}$ ranges from $\sim 1$ to $\sim 6$ events when assuming a cut-off energy of $2.2 \mathrm{PeV}$ and $100 \mathrm{PeV}$, respectively.

\section{Conclusion and outlook}

A new method to search for PeV events using partially-contained cascades has been introduced. It has been optimised to complement the existing search methods. It increases the effective area at the energy of the Glashow resonance by a factor of $\sim 2$, where the expected number of events varies strongly depending on the assumption of the spectral shape. Soon 6-years of full data will be unblinded and a global-fit for a cut-off including HESE, EHE-PeV and PEPE samples will be performed. Systematic uncertainties such as the optical properties of ice and hadronic interaction models for atmospheric muon production will be included.

\section{References}

[1] IceCube Collaboration, M. G. Aartsen et al., Phys. Rev. Lett. 111 (2013) 021103

[2] IceCube Collaboration, M. G. Aartsen et al., Science 342 (2013) 1242856

[3] IceCube Collaboration, M. G. Aartsen et al., Phys. Rev. Lett. 113 (2014) 101101

[4] IceCube Collaboration, M. G. Aartsen et al., Astrophys.J. 833 (2016) 3

[5] S. L. Glashow, Phys. Rev. 118 (1960) 316

[6] V. Barger et al., Phys. Rev. D 90 (2014) 121301

[7] IceCube Collaboration, M. G. Aartsen et al., Astropart. Phys. 92 (2017) 30

[8] IceCube Collaboration, M. G. Aartsen et al., PoS(ICRC2015) 1109 (2016)

[9] Y. Freund and R. E. Schapir, J. Comput. Syst. Sci. 55 (1997) 1-119-139

[10] D. Heck et al., Technical Report, Forschungszentrum Karlsruhe GmbH 6019 (1998)

[11] F. Riehn et al., PoS(ICRC2015) 558 (2016)

[12] IceCube Collaboration, M. G. Aartsen et al., Proceedings of ICRC2013 0580 (2014)

[13] G. Cowan et al., Eur.Phys.J. C71 (2001) 1554

[14] IceCube Collaboration, M. G. Aartsen et al., PoS(ICRC2015) 1066 (2016)

[15] S. S. Wilks, Ann. Math. Statist. 9 (1938) 1-60-62 